\title{
Alopecia in general medicine
}

\author{
Authors: Rajani Nalluri ${ }^{A}$ and Matthew Harries ${ }^{\mathrm{B}}$
}

\begin{abstract}
Appreciation of different types of hair loss (alopecia) that may be encountered in hospital medicine is important to ensure accurate diagnosis and management, identify underlying medical conditions or treatments that may present with increased hair loss, recognise autoimmune alopecias and their associations, and understand the significant psychological impact of hair loss on an individual. This article discusses common causes of hair loss, as well as those conditions that may be associated with systemic disease, relevant to a general physician.
\end{abstract}

\section{Introduction}

Hair loss (alopecia) is a relatively common feature encountered in general medicine. The cause of the problem is often quickly apparent when careful assessment is undertaken, allowing explanation and appropriate therapy. However, hair loss can sometimes be the presenting feature of a significant underlying medical condition, may be seen in association with other diseases, or may be a side effect of a therapy. ${ }^{1}$ Further, some conditions display significant and distressing symptoms, while others might result in permanent scarring alopecia. Thus, accurate diagnosis and prompt treatment is paramount in these disorders. Here we discuss common causes of hair loss, with specific emphasis on those conditions encountered by a general physician.

\section{The psychological impact of hair loss}

Frequently overlooked by healthcare professionals is the significant psychological distress that occurs when people start to lose their hair. This may be, in part, due to the cosmetic impact of visible hair loss and fear of becoming completely bald, but also about the significance of this problem to their general health. Many sufferers experience feelings of distorted self-image and low self-esteem and may display symptoms of anxiety and depression. ${ }^{2}$

Authors: ${ }^{\text {A }}$ specialist trainee in dermatology, The University of Manchester, Salford Royal NHS Foundation Trust, Salford, UK; ${ }^{B}$ consultant dermatologist and honorary senior lecturer, The University of Manchester, Salford Royal NHS Foundation Trust, Salford, UK

\section{Hair follicles, the hair cycle and hair loss}

Hair is a defining feature of mammals; important in thermoregulation, proprioception and camouflage. Each hair on our body is constantly undergoing cycles of growth (anagen), regression (catagen), resting (telogen) and shedding (exogen), with each hair cycling independently from its neighbour. ${ }^{3}$ When hair loss occurs it is usually due to either a hair cycle problem (ie a change in the proportion of hairs in, or length of, each phase), or due to inflammation directly affecting the hair follicle (HF). When the epithelial HF stem cells located at the insertion of the arrector pili muscle ('bulge region') are damaged by inflammation the hair can no longer cycle or regenerate resulting in HF loss and permanent ('scarring') alopecia (Fig 1).

\section{Increased hair shedding (telogen effluvium)}

Telogen effluvium is one of the commonest causes of hair loss that presents as increased hair shedding and diffuse thinning 1- 6 months after a triggering event. The scalp skin looks normal with no inflammation or scarring present. Increased numbers of telogen hairs can be easily removed when the hair is pulled. Telogen effluvium results from an increased proportion of hairs prematurely entering the telogen (resting) phase of the growth cycle before they are subsequently shed. Acute or chronic illness, surgery, changing medication, thyroid dysfunction, nutritional deficiency and severe stress are common triggers (see Table 1). ${ }^{1,4}$ A careful history is required to identify any potential triggering factors or underlying conditions. If no apparent cause is identified screening blood tests are usually performed to identify an occult underlying disorder (Table 1). ${ }^{4}$ Once the potential cause is identified and treated, regrowth should occur within 6 months.

\section{Chemotherapy hair loss (anagen effluvium)}

Highly proliferating cells in the hair bulb that produce the hair shaft are particularly susceptible to chemotherapy agents. Anagen effluvium occurs when anagen (growing) hairs stop growing abruptly, resulting in a weakened hair shaft that easily breaks at the skin surface. Hair loss is usually severe and extensive as $>85 \%$ hairs at any one time are in the anagen growth phase. $^{5}$

Chemotherapy-induced alopecia (CIA) can be anticipated depending on the regimen used and so is usually managed with psychological support and wig provision. Scalp cooling during chemotherapy can reduce HF metabolisms and reduce hair loss 


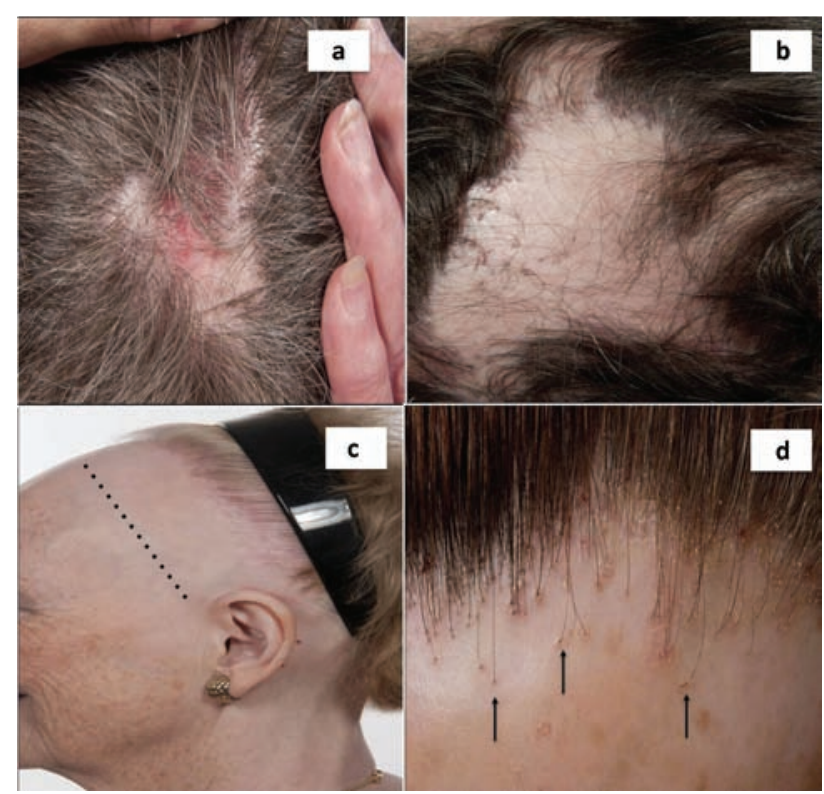

Fig 1. Scarring alopecia. a) Chronic cutaneous lupus erythematosus: scarring hair loss with erythema and scale in the centre of the patch. Examine the rest of the skin for other similar lesions; b) LPP: scarring hair loss on the central scalp with inflammation at the edge of the patch. Close inspection reveals perifollicular erythema and scale at the periphery of the patch; $c$ ) FFA: progressive ('band-like') frontal hairline recession with loss of eyebrows (dotted line represents the original hairline); d) FFA: close-up view reveals loss of visible follicular openings, perifollicular erythema and perifollicular scale. Vellus hairs are no longer visible and 'lonely hairs' (ie hairs that remain while others around them have been lost) can be seen (arrows). The same features shown here can also be seen in LPP. FFA = frontal fibrosing alopecia; LPP = lichen planopilaris.

in some patients, although this may not be suitable for all cancer types. ${ }^{6}$ Interestingly, a study in breast cancer chemotherapy showed that application of topical 2\% minoxidil lotion applied twice a day during therapy and for 4 months after significantly reduced the time to complete hair regrowth by a mean of 50.2 days ( 86.7 minoxidil vs 137.9 placebo). ${ }^{7}$ In total, $47 \%$ of people cite hair loss as the most traumatic side effects of chemotherapy with $8 \%$ rejecting treatment due to fear of alopecia. ${ }^{8}$

Chemotherapy-induced hair loss is generally reversible, ${ }^{5}$ although there is a growing recognition that in rare cases certain regimens can result in permanent alopecia. Permanent CIA is defined as incomplete regrowth $>6$ months after completion of treatment and likely results from irreversible HF stem cell damage. Hair loss is usually diffuse, incomplete and non-inflamed with more marked thinning over the vertex reminiscent of androgenetic alopecia (Fig 2). ${ }^{9}$ Awareness of permanent CIA is needed so patients can be adequately counselled before therapy. This is particularly important for newer breast cancer adjuvant regimens containing taxanes (eg docetaxel or paclitaxel), as litigation may result without appropriate informed consent. ${ }^{10}$

\section{Female and male pattern hair loss}

Female pattern hair loss (FPHL) is a slowly progressive, nonscarring alopecia characterised by diffuse thinning over the

Table 1. Causes and investigations for telogen effluvium.

\section{Causes of telogen} effluvium

\section{Acute illness \\ Febrile illness \\ Blood loss/trauma \\ Chronic illness \\ Thyroid dysfunction \\ Hepatic / renal failure \\ HIV / Syphilis \\ Malignancy \\ Nutritional deficiency \\ Protein-calorie \\ malnutrition \\ Iron/zinc/vitamin D \\ deficiency \\ Malabsorption \\ Hormonal changes \\ Stop/start/or change COCP}

\section{Medications}

'Anti-drugs' (eg anti-coagulants; antidepressants; antiarrhythmic; anti-thyroid)

Retinoids

\section{Beta-blockers}

\section{Heavy metal poisoning}

Arsenic, thallium

\section{Severe emotional stress}

\section{Early androgenetic} alopecia

$\mathrm{ANA}=$ antinuclear antibody; $\mathrm{COCP}=$ combined oral contraceptive pill; $\mathrm{ESR}=$ erythrocyte sedimentation rate; $F B C=$ full blood count; TFT = thyroid function test.

crown and mid-frontal scalp with preservation of the frontal hairline. A widening of the midline hair part (Fig 3) with preservation of density over the occipital scalp are characteristic features. ${ }^{11}$ Hair loss occurs due to miniaturisation of terminal hairs to vellus hairs, reduction in anagen duration and longer gaps between hair cycles when the follicle is empty (kenogen) in genetically susceptible HFs. Trichoscopy (scalp dermoscopy) shows variability of hair shaft diameter and increased proportion of vellus hairs. Topical $2 \%$ minoxidil lotion is the only licensed treatment for FPHL currently available.

The role of excessive androgens causing FPHL is controversial. ${ }^{12}$ Certainly a small proportion of women with FPHL do have an abnormal androgen profile, although most do not. Thus, anti-androgen therapy (eg spironolactone/ finasteride) commonly used off licence in FPHL often shows 


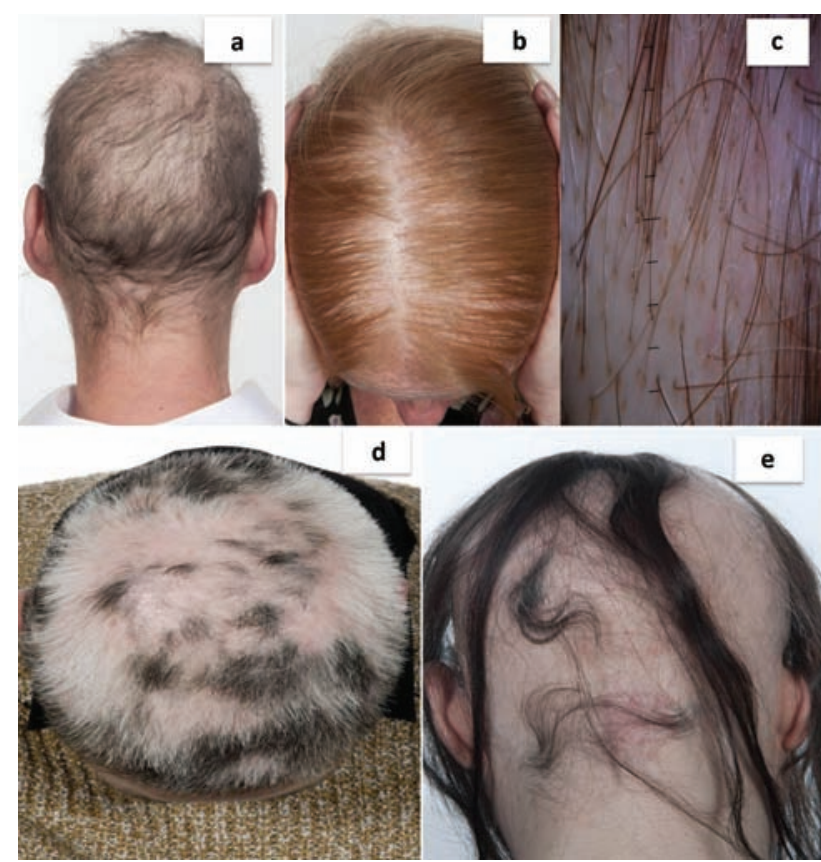

Fig 2. Non-scarring alopecia. a) Permanent chemotherapy-induced alopecia: diffuse non-inflamed hair loss; b) FPHL: widened hair part with preservation of the frontal hair line. Close inspection reveals variation of hair shaft diameter and increased proportion of (miniaturised) vellus hairs; c) Trichoscopy image (magnification, x10) of FPHL showing hair shaft thickness diversity, multiple thin vellus hairs, increased proportion of single hair follicular units and numerous yellow dots (due to accumulation of sebum at the follicular opening); d) alopecia areata: patchy hair loss with white hair regrowth. These white hairs should repigment with time; e) alopecia areata: more extensive hair loss with no inflammation or scarring. $\mathrm{FPHL}=$ female pattern hair loss.

an inconsistent response. Screening blood tests (testosterone/ sex hormone binding globulin/prolactin) are recommended in women with rapidly progressive or 'male pattern' of hair loss (see below), or if they display any features to suggest hyperandrogenism, including acne, seborrhoea, hirsutism, menstrual irregularities or signs of virilisation. ${ }^{13}$ Determining the cause of abnormal androgens (eg polycystic ovarian syndrome, congenital adrenal hyperplasia, androgen-secreting tumour and Cushing's syndrome) may require more extensive endocrine investigations and specialist referral.

Male pattern hair loss predominantly affects the crown and temples progressing to complete baldness. HF sensitivity to androgens drives the miniaturisation process. Licensed treatments include topical minoxidil and the $5 \alpha$-reductase inhibitor finasteride (Propecia ${ }^{\circledR}$ ). Sexual side effects (loss of libido and erectile dysfunction) are associated with finasteride therapy and may even persist on stopping treatment.

\section{Alopecia areata}

Alopecia areata (AA) is an autoimmune T-cell-mediated non-scarring alopecia with an estimated lifetime risk of $1.7 \%$. The classic presentation is of sudden-onset completely bald patches with increased hair fall. ${ }^{14}$ Dermatoscopic features include yellow dots and exclamation mark hairs (short broken hairs with a broad distal end seen along the edges of the patches that are a marker of disease activity). Most sufferers have one or more circular patches of hair loss, although extensive or complete hair loss may occur (ie alopecia totalis/universalis). Nails may be involved in $10 \%$ of patients with pitting and trachyonychia being the most common abnormalities. ${ }^{15}$ Histology reveals a dense T-cell infiltrate around the HF bulb ('swarm of bees'). This interrupts hair growth by converting the HF from anagen to catagen thereby precipitating hair loss. Future hair cycles may also be affected with anagen follicles forced into catagen before the hair shaft has fully formed. AA often runs a relapsing and remitting course but with a trend towards more extensive hair loss over time. ${ }^{16}$

AA is associated with atopic and other autoimmune disorders including vitiligo, lupus erythematosus (LE), psoriasis, thyroid disease and atopic eczema. Interestingly, the age of presentation of AA affects the risk of developing comorbidities, with AA onset before 10 years linked with higher risk of LE and atopic eczema, and AA onset after 60 years associated with thyroid disease. $^{17}$

A recent genome-wide association study (GWAS), involving 1,054 AA cases and 3,278 controls, identified 139 single nucleotide polymorphisms with susceptibility loci clustered in 8 genomic regions, implicated in innate and adaptive immune functioning. ${ }^{18}$ Of particular interest were loci on chromosome 2q33.2 containing CTLA4, chromosome 6p21.32 HLA genes and chromosome 6q25.1 harbouring the ULBP genes. These ULBP genes function as natural killer group $2 \mathrm{D}$ (NKG2D)-activating ligands in the hair bulb that mediate recruitment of $\mathrm{CD} 8{ }^{+} \mathrm{NKG} 2 \mathrm{D}^{+} \mathrm{T}$ lymphocytes necessary for disease development. ${ }^{18}$ Interestingly, the GWAS identified clear commonality in susceptibility loci between AA and other autoimmune conditions, opening the possibility of realigning drugs used in these disorders to also treat AA. The two main foci of interest are the rheumatoid arthritis drug abatacept, a recombinant CTLA4-Ig that blocks co-stimulatory signals preventing T-cell activation, and the Janus kinase (JAK) inhibitors ruxolitinib and tofacitinib. ${ }^{19}$
Fig 3. Sinclair scale: 5-point scale for female pattern hair loss with diffuse thinning of hair on the crown and widening of the midline hair part with preservation of the frontal hairline. Reproduced with permission. $^{11}$

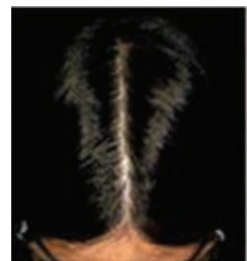

1

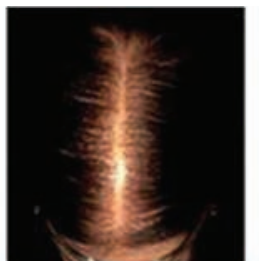

2

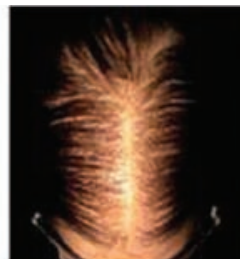

3

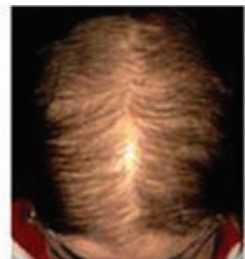

4

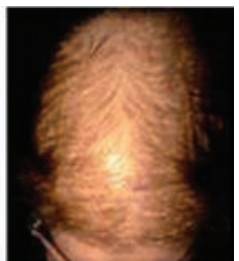

5 


\section{Janus kinase inhibitors - a promising new treatment}

JAK inhibitors act by blocking downstream effects of proinflammatory cytokines (eg interferon- $\gamma$ ) and interleukin-15 thereby preventing T-cell activation. In mice models JAK inhibitors have been shown to prevent the development of AA and reduced $\mathrm{CD} 8{ }^{+} \mathrm{NKG} 2 \mathrm{D}^{+}$cell numbers. ${ }^{20}$ Limited reports in humans also suggest a beneficial effect. ${ }^{20,21}$ Significant treatment costs and side-effect concerns currently limit uptake of this therapy in the UK.

\section{Scarring alopecias}

Although uncommon, scarring (cicatricial) alopecias are important to recognise as they are frequently symptomatic and result in permanent hair loss. They are characterised clinically by loss of visible follicular ostia with variable degrees of inflammation. ${ }^{22}$ HF stem cells in the bulge region are damaged and destroyed by inflammation with subsequent HF loss and replacement with scar-like fibrous tissue. ${ }^{23}$ Primary scarring alopecias occur when the HF itself is targeted, whereas secondary scarring alopecias occur when HFs are caught up in a more generalised damaging process within the skin (eg sarcoidosis, morphoea, thermal burns and ionizing radiation). The diagnosis should be confirmed by scalp biopsy. ${ }^{24}$ Treatment approaches are generally aimed at suppressing inflammation to prevent further hair loss and control symptoms. Therapies include topical and intra-lesional corticosteroids, tetracycline antibiotics, anti-malarials and systemic immunosuppression. ${ }^{25}$

\section{Lichen planopilaris}

Lichen planopilaris (LPP) is thought to be a follicular variant of lichen planus and is the commonest primary scarring

\section{Key points}

Hair loss usually results either from alterations to the hair growth cycle or from inflammatory damage to the hair follicle.

Increased hair shedding (telogen effluvium) may signal an underlying medical problem and should be investigated appropriately.

Chemotherapy may rarely result in permanent hair loss with taxane-containing regimens for breast cancer being recently implicated.

A number of autoimmune conditions have similar genetic susceptibilities in common with alopecia areata and this could pave the way for new treatment development.

Scarring alopecias may result from a number of underlying conditions. Scalp biopsy is often required to confirm the diagnosis.

KEYWORDS: Alopecia, hair loss, chemotherapy, JAK inhibitors, autoimmune alopecia seen. Clinical features include patchy hair loss on the central scalp, with visible scarring, perifollicular erythema and perifollicular scale. Inflammation is seen at the edge of the patches. Itch and tenderness is common and lichen planus at another skin site, including the mucous membranes or nails, may occur in up to $20 \%$ patients. Over recent years a variant of LPP called frontal fibrosing alopecia (FFA), first described only 20 years ago, appears to be rapidly increasing in incidence. Here progressive frontal hairline recession associated with eyebrow and body hair loss occurs. It predominantly affects post-menopausal women, although pre-menopausal women and men are now being recognised with the condition. Why more cases of FFA are presenting is unclear but may represent an (as yet unknown) environmental factor in disease aetiology.

\section{Chronic cutaneous lupus erythematosus}

Chronic cutaneous lupus erythematosus (CCLE; discoid lupus) presents as erythematosus, scaly plaques that can involve the body and scalp. Close inspection reveals patchy hair loss, scarring, follicular plugging, atrophy and dyspigmentation with inflammation seen in the centre of the plaques. Evidence of systemic lupus erythematosus (SLE) should be sought with careful history, examination and autoantibody screening, although the risk of SLE in patients presenting with CCLE is generally low. Strict ultraviolet light protection and prompt treatment can minimise scarring and may, in rare cases, result in hair regrowth.

\section{Other conditions causing hair loss}

Scalp fungal infections in adults are uncommon, but can sometimes mimic other conditions, including scarring alopecias. Always send samples for microbiology assessment in any inflammatory patchy hair loss where increased scale, broken hairs or pustules are present.

Secondary syphilis can present with patchy 'moth-eaten' alopecia that is non-scarring. The patient is often unwell with malaise, fever, lymphadenopathy and a non-itchy papulosquamous rash. Check syphilis serology, particularly if risk factors for infection are identified.

Sarcoidosis is a multisystem disorder that affects the skin, and rarely the scalp, causing scarring alopecia. Papules, nodules and plaques may be present elsewhere on the skin and characteristically involve scars. Diagnosis is made with skin biopsy.

Linear morphoea ('en coup de sabre') involves the forehead and scalp, resulting in a unilateral linear sclerotic hairless plaque; it is sometimes associated with hemi-facial atrophy. Scalp skin metastases may present with scalp nodules, whereas alopecia neoplastica (ie neoplasm directly involving the HF) may present with one or more alopecia patches that can resemble AA or scarring alopecia. Breast cancer is the commonest cause. Diagnosis is made with scalp biopsy.

Follicular mucinosis described the histological feature of mucinous degeneration of the HF epithelium most commonly seen in cutaneous T-cell lymphoma/follicular mycosis fungoides. Infiltrated plaques and follicular papules with associated alopecia are characteristic, particularly when the eyebrows are involved. 


\section{Conclusions}

We present a number of hair loss disorders that may be encountered by a general physician. We hope that readers are now more aware of associated comorbidities, recognise that alopecia may be a marker for an underlying medical condition, identify conditions that can result in permanent scarring hair loss and appreciate the significant psychological impact. Accurate diagnosis is essential so we can provide information to our patients and offer appropriate therapy. A greater understanding of the pathobiology of all these disorders will help identify novel targets for therapy. Lessons learned from related conditions should hasten development of new therapies, with the re-alignment of existing treatments being a promising avenue for research.

\section{References}

1 Macbeth A, Harries M. Hair loss in hospital medicine: a practical guide. Br J Hosp Med (Lond) 2012;73:372-9.

2 Hunt N, McHale S. The psychological impact of alopecia. BMJ 2005;331:951-3.

3 Paus R, Cotsarelis G. The biology of hair follicles. N Engl J Med 1999;341:491-7.

4 Cunningham C, Paus R, Harries M. Recurrent episodes of hair loss in a 37 year old woman. BMJ 2012;345:e6798.

5 Kanti V, Nuwayhid R, Lindner J et al. Analysis of quantitative changes in hair growth during treatment with chemotherapy or tamoxifen in patients with breast cancer: a cohort study. $\mathrm{Br} J$ Dermatol 2014;170:643-50.

6 Shin $\mathrm{H}$, Jo SJ, Kim do $\mathrm{H}$ et al. Efficacy of interventions for prevention of chemotherapy-induced alopecia: a systematic review and meta-analysis. Int J Cancer 2015;136:E442-54.

7 Duvic M, Lemak NA, Valero V et al. A randomized trial of minoxidil in chemotherapy-induced alopecia. J Am Acad Dermatol 1996;35:74-8.

8 McGarvey EL, Baum LD, Pinkerton RC et al. Psychological sequelae and alopecia among women with cancer. Cancer Pract 2001;9:283-9.

9 Miteva M, Misciali C, Fanti PA et al. Permanent alopecia after systemic chemotherapy: a clinicopathological study of 10 cases. Am J Dermatopathol 2011;33:345-50.

10 Tosti A, Palamaras I, Miteva M et al. Docetaxel and permanent alopecia. J Am Acad Dermatol 2013;68:e151.

11 Biondo S, Goble D, Sinclair R. Women who present with female pattern hair loss tend to underestimate the severity of their hair loss. Br J Dermatol 2004;150:750-2.
12 Messenger AG. Hair through the female life cycle. Br J Dermatol 2011;165:2-6.

13 Blume-Peytavi U, Blumeyer A, Tosti A et al. S1 guideline for diag nostic evaluation in androgenetic alopecia in men, women and adolescents. Br J Dermatol 2011;164:5-15.

14 Harries MJ, Sun J, Paus R et al. Management of alopecia areata. BMJ 2010;341:c3671.

15 Messenger AG, McKillop J, Farrant P et al. British Association of Dermatologists' guidelines for the management of alopecia areata 2012. Br J Dermatol 2012;166:916-26.

16 Tosti A, Bellavista S, Iorizzo M. Alopecia areata: a long term follow-up study of 191 patients. J Am Acad Dermatol 2006;55:438-41.

17 Chu SY, Chen YJ, Tseng WC et al. Comorbidity profiles among patients with alopecia areata: the importance of onset age, a nationwide population-based study. J Am Acad Dermatol 2011;65:949-56.

18 Petukhova L, Duvic M, Hordinsky M et al. Genome-wide association study in alopecia areata implicates both innate and adaptive immunity. Nature 2010;466:113-7.

19 Norris DA. Genes and immune response in alopecia areata: review of the alopecia areata research summit first day proceedings. $J$ Investig Dermatol Symp Proc 2013;16:S10-2.

20 Xing L, Dai Z, Jabbari A et al. Alopecia areata is driven by cytotoxic T lymphocytes and is reversed by JAK inhibition. Nat Med 2014;20:1043-9.

21 Craiglow BG, King BA. Killing two birds with one stone: oral tofacitinib reverses alopecia universalis in a patient with plaque psoriasis. J Invest Dermatol 2014;134:2988-90.

22 Harries MJ, Trueb RM, Tosti A et al. How not to get scar(r)ed: pointers to the correct diagnosis in patients with suspected primary cicatricial alopecia. Br J Dermatol 2009;160:482-501.

23 Harries MJ, Meyer K, Chaudhry I et al. Lichen planopilaris is characterized by immune privilege collapse of the hair follicle's epithelial stem cell niche. J Pathol 2013;231:236-47.

24 Olsen EA, Bergfeld WF, Cotsarelis G et al. Summary of North American Hair Research Society (NAHRS)-sponsored Workshop on Cicatricial Alopecia, Duke University Medical Center, February 10 and 11, 2001. J Am Acad Dermatol 2003;48:103-10.

25 Harries MJ, Sinclair RD, Macdonald-Hull S et al. Management of primary cicatricial alopecias: options for treatment. Br J Dermatol 2008;159:1-22.

Address for correspondence: Dr M Harries, The Dermatology Centre, The University of Manchester, Salford NHS Foundation Trust, Salford M6 8HD, UK.

Email: matthew.harries@srft.nhs.uk 